\title{
Avaliação de Políticas Públicas de Turismo: uma análise bibliométrica dos periódicos de turismo
}

\section{Evaluation of Public Politics of Tourism: a bibliometrical analysis of tourism periodic}

\section{Evaluación de Politicas Públicas de Turismo: un análisis bibliométrica de los periódicos de turismo}

\author{
Alba Oliveira Barbosa Lopes ${ }^{1}$ \\ Dinah dos Santos Tinôco ${ }^{2}$ \\ Lincoln Moraes de Souza ${ }^{3}$
}

\begin{abstract}
Resumo
Este trabalho tem por objetivo fazer um levantamento dos artigos que versam sobre avaliação de políticas públicas em turismo nos periódicos especializados, avaliados e classificados pela Coordenação de Aperfeiçoamento de Pessoal de Nível Superior-CAPES com conceitos iguais ou superiores a B4. Quanto à metodologia, foram selecionados artigos cujos títulos apresentaram uma ou mais palavras-chaves referentes à avaliação de política pública de turismo no período 2003-2010. O intuito foi de elucidar a freqüência da produção acadêmica sobre avaliação, autores e instituições, os objetos dos estudos, procedimentos metodológicos e citações da bibliografia especializada. Para tanto, utilizou-se da técnica de análise de conteúdo. Os resultados mostraram que é irrelevante o lugar e o espaço ocupado pela avaliação de políticas públicas de turismo nos principais periódicos de turismo. Verificou-se diversidade de autores e instituições e pouca utilização da bibliografia especializada, tanto sobre avaliação quanto sobre turismo. A conclusão destacou a falta de clareza quanto à metodologia utilizada nos artigos, principalmente em relação às categorias analíticas e indicadores, o que comprometeu parte das análises e principais conclusões.
\end{abstract}

Palavras-chave: bibliometria; avaliação de políticas públicas; turismo.

\footnotetext{
${ }^{1}$ Doutoranda em Administração pela UFRN-Universidade Federal do Rio Grande do Norte. Graduada e mestre em Administração pela UFPE-Universidade Federal de Pernambuco. Professora do IFPE-Instituto Federal de Pernambuco. e-mail: albabarbosa@ig.com.br

2 Pós-doutora pela Universidade de Versailles Saint Quentin, França. Doutora e mestre em Urbanismo pela Universidade de Paris XII. Graduada em Economia pela UFRN onde é Professora Associada no Departamento de Ciências Administrativas. E-mail: dinahtinoco@uol.com.br

${ }^{3}$ Doutor em Política Social pela Universidade Estadual de Campinas. Mestre em Sociologia pela Universidade de Brasília. Graduado em Ciências Sociais pela Universidade Federal do Ceará. Professor Adjunto da UFRN, no Departamento de Ciências Sociais. E-mail: lincolnmoraes@ufrnet.br
} 


\begin{abstract}
This work has the objective to gather de articles that talk about public politics evaluation in specific periods, evaluated and classified by Coordination of Improvement of Personal with CAPES Upper Level with the same concepts or higher to B4. As for the methodology, there have been selected articles of which the titles presented one or more key words concerning to the evaluation of public politics of tourism in the period of 2003-2010. They attempted to elucidate the frequency of academic production of evaluation, authors and institutions, the objects of studies, methodological procedures and quotes of specialized bibliography. For that, an analysis technique of contents was used. The results showed that time and space occupied for the evaluation of public politics of tourism is irrelevant in the main periods of tourism. Diversity was verified of authors and institutions and little use of the specialized bibliography, both for evaluation and tourism. Conclusion highlighted the lack of clarity as for the methodology utilized in the articles, especially regarding to analytical categories and indicators, which compromised part of the analysis and main conclusions.
\end{abstract}

Keywords: bibliometry; evaluation of public politics; tourism.

\title{
Resumen
}

Este trabajo tiene por objetivo hacer un levante de los articulos que versan sobre evaluación de políticas publicas en turismo en los periódicos especializados, evaluados y clasificados por la Coordinación de Mejora de personal de Nivel Superior - CAPES con conceptos iguales o superiores a B4. Cuanto la metodología fueron seleccionados artículos cuyos temas presentaron una o más palabras-claves referentes la evaluación de politicas publicas de turismo en lo periodo de 2003-2010. La finalidad fue de elucidar la frecuencia de la producción académica sobre evaluación, autores e instituciones, los objetos de los estudios, procedimientos metodológicos y citaciones de la bibliografía especializada. Por lo tanto, se utiliza la técnica de análisis de contenido. Los resultados mostraban que es irrelevante el sitio o el espacio ocupado por la evaluación de políticas publicas de turismo en los principales periódicos de turismo. Se detecto la diversidad de autores e instituciones y poca utilización de la bibliografía especializada, tanto sobre evaluación cuanto sobre turismo. La conclusión se destacó por la falta de claridad cuanto la metodología usada en los artículos, principalmente en relación a las categorías analíticas e indicadores, lo que comprometió parte de los análisis y de las principales finalizaciones.

Palabras-clave: bibliometria; evaluación de politicas publicas; turismo. 


\section{TURISMO EM ANÁLISE}

\section{Introdução}

Este artigo apresenta informações de um levantamento inicial com o objetivo de subsidiar uma pesquisa de doutoramento na área de avaliação de políticas públicas de turismo. O intento foi identificar autores, obras e instituições de referência, bem como tipologias e metodologias de avaliação utilizadas nos estudos publicados nos periódicos de turismo avaliados pela Coordenação de Aperfeiçoamento de Pessoal de Nível Superior (CAPES). Este artigo segue a tendência apontada por Caldas e Tinoco (2004) que observaram a proliferação de artigos científicos sobre análise da produção acadêmica nacional em diversas áreas.

O estudo das políticas públicas de turismo tem ampliado sua importância devido ao crescimento da atividade nos países em virtude de motivações diversas. O turismo tem sido divulgado como uma alternativa para desenvolver econômica e socialmente as localidades, no entanto, esse desenvolvimento pode não ocorrer, e não é difícil encontrar casos de crescimento turístico que também foram geradores de irreversíveis problemas ambientais e aumento da concentração de renda.

A política de turismo pode ser entendida como um conjunto de ações de entes governamentais, muitas vezes em parcerias com outros entes, com vistas a modificar o meio econômico e social. A política, em geral, apresenta as diretrizes básicas da atividade nos países onde se encontra, os objetivos gerais e as prioridades de ação estatal para o setor.

Em princípio, a política pública de turismo deveria atender aos seguintes requisitos: a) definir os termos nos quais as operações turísticas devem funcionar; b) estabelecer códigos de conduta e de ética a serem respeitados pelos visitantes, fornecedores e comunidade; c) facilitar o diálogo em torno de estratégias e objetivos específicos para uma destinação; d) promover uma pauta de discussões com agentes públicos e privados sobre o papel e as contribuições do setor e e) permitir a interface do turismo com outros setores econômicos (GOELDNER, RITCHIE, McINTOSH, 2002).

A atuação estatal no turismo não é necessariamente benéfica ou prejudicial. Conforme Brohman (1996), não existe nada de errado em aumentar o apoio do Estado para os novos setores de crescimento voltados para fora, como no caso do turismo, notadamente o 


\section{TURISMO EM ANÁLISE}

internacional. No entanto, depende da maneira que o turismo atende às necessidades e interesses da população local e como ele se encaixa nas metas de desenvolvimento social. As autoras desse artigo acreditam que ao se estudar o processo de avaliação das políticas, programas ou projetos na área do turismo poderia ser direcionado esforços na condução mais efetiva dessas ações estatais, especialmente nos processos de formulação e implementação das políticas.

\section{Avaliação de Políticas Públicas}

Nessa seção serão apresentados alguns conceitos de autores relevantes na área de Avaliação de Políticas Públicas. Em seguida, é explicitada a metodologia que serviu de base para o trabalho de avaliação bibliométrica da área de turismo.

A pesquisa de avaliação tem, para Draibe (2001, p. 17), como objeto "uma dada intervenção na realidade social, uma política pública, entendida então como a que se desenvolve em esferas públicas da sociedade". Esse entendimento norteou a seleção dos artigos a serem investigados nesse trabalho, ou seja, foram selecionados artigos que contemplassem intervenções de entes governamentais na realidade social.

A avaliação de políticas públicas passou a ter maior destaque nas últimas décadas, atraindo pesquisadores e gestores públicos para o desenvolvimento de técnicas e métodos de avaliação (CAIDEN; CAIDEN, 2001; PINTO, 1989). Até a década de 70, dominava a avaliação baseada em instrumentos econométricos que foram sendo objetos de questionamentos à medida que a avaliação torna-se um campo multidisciplinar. A avaliação sob a perspectiva qualitativa torna-se interesse de muitos pesquisadores desde então, enfatizando, dentre outros aspectos, a participação e percepção dos entes envolvidos nas diversas etapas da politica social (formulação, implementação e avaliação).

Minayo (2005) enfatiza que os seguintes fatores contribuíram para o crescimento do interesse e das práticas avaliativas: a) reforma do Estado, que incentivou o crescimento de seu papel de formulador de políticas e fornecedor de recursos para o terceiro setor; b) focalização da ação do governo em atividades setoriais, exigindo análise de eficácia dos investimentos; c) 


\section{TURISMO EM ANÁLISE}

exigências de organismos financiadores internacionais por maiores e melhores avaliações; d) ampliação de mecanismos de controle pela sociedade, através de vigilância e instrumentos de pressão da sociedade.

Figueiredo e Figueiredo (1986) montaram um quadro de referências teóricas sobre a avaliação de políticas setoriais que continua sendo bastante útil quase 25 anos depois. Em primeiro lugar, eles estabelecem a diferença entre avaliação política e avaliação de políticas. A primeira pode ser vista, segundo os autores, como uma etapa preliminar para a segunda, onde se analisa e elucida o critério ou critérios que a tornaram preferível a qualquer outra política. A avaliação política não avalia a efetiva apropriação dos benefícios da política por parte da população. A segunda busca identificar o sucesso ou o fracasso de uma determinada intervenção social. Esse sucesso ou fracasso, logicamente, depende dos objetivos da política e das motivações do analista. A última, em geral incorpora a avaliação da efetiva apropriação dos benefícios pela população-alvo da política.

A avaliação de políticas pode: a) ser uma atividade que obtém, combina e compara dados de desempenho com um conjunto de metas, b) responder a questões sobre eficácia/efetividade dos programas com a finalidade de julgar e informar e c) detectar falhas e aferir méritos dos programas durante a sua elaboração para permitir correção ou confirmação de rumos (FARIA, 2007). A avaliação de políticas públicas pode ser definida como,

(...) uma forma de pesquisa social aplicada, sistemática, planejada e dirigida; destinada a identificar, obter e proporcionar de maneira válida e confiável dados e informação suficiente e relevante para apoiar um juízo sobe o mérito e o valor dos diferentes componentes de um programa (tanto na fase de diagnóstico, programação ou execução), ou de um conjunto de atividades específicas que se realizam, foram realizadas ou se realizarão; comprovando a extensão e o grau em que se deram essas conquistas, de forma tal que sirva de base ou guia para uma tomada de decisão racional e inteligente entre cursos de ação, ou para solucionar problemas e promover o conhecimento e a compreensão dos fatores associados ao êxito ou ao fracasso de seus resultados (AGUILAR; ANDER-EGG, 1994, p. 31-32). 


\section{TURISMO EM ANÁLISE}

A definição citada acima expõe que a avaliação utiliza-se de procedimentos baseados no método científico para identificar informações que possibilitará a emissão de julgamentos acerca das políticas, programas ou projetos. Os autores destacam que além das ações formalmente estabelecidas, também podem ser avaliados atividades específicas que se organizam para conseguir um determinado objetivo. Essas análises servirão para otimizar o processo de tomada de decisões e devem prestar contas dos fatores que contribuirão para o êxito ou fracasso de uma ação.

\subsection{Mensuração de políticas públicas: o papel dos indicadores}

Os indicadores sociais fornecem subsídios para a atividade de planejamento público e formulação de políticas sociais ao possibilitar o monitoramento das condições de vida e bemestar da população. Os indicadores são medidas que operacionalizam um conceito abstrato e permitem o aprofundamento da investigação acadêmica (JANUZZI, 2005). Ainda segundo o autor, este "é uma medida, em geral, quantitativa dotada de significado social substantivo, usado para substituir, quantificar ou operacionalizar um conceito social abstrato, de interesse teórico (para pesquisa acadêmica) ou programático (para formulação de políticas)" (JANUZZI, 2002, p. 54).

De acordo com Januzzi (2002, 2002a, 2005), a escolha dos indicadores a serem utilizados no processo de avaliação de políticas públicas depende da finalidade a que eles se destinam e a fase do processo de formulação e implementação da política social. Eles podem ser classificados segundo:

a) à área temática da realidade social a que se referem: indicadores de saúde, educacionais, mercado de trabalho, indicadores demográficos, habitacionais, segurança pública e justiça, de infraestrutura urbana, de renda e desigualdade (CARLEY, 1985 apud JANUZZI 2002a).

b) à natureza do ente indicado: indicador-insumo, indicador-processo, indicador-resultado, indicador-impacto. Os indicadores-insumo são medidas que estão associados à disponibilidade de recursos alocados ao programa ou projeto analisados. Os indicadoresprocesso traduzem o esforço operacional de alocação de recursos para a obtenção de melhorias efetivas de bem-estar. Os indicadores-resultados estão vinculados aos objetivos 


\section{TURISMO EM ANÁLISE}

finais dos programas públicos e permitem avaliar a eficácia dos programas. Os indicadoresimpacto referem-se aos efeitos mais gerais da implantação dos programas (OMS, 1996 apud JANUZZI 2002a, 2005).

c) Aspectos relevantes da avaliação de programas sociais: indicadores para avaliação de eficiência dos meios e recursos empregados, indicadores para avaliação da eficácia no cumprimento das metas e indicadores para avaliação da efetividade social do programa. (CARDOSO, 1999 apud JANUZZI, 2002, 2002a).

Antes de finalizar essa seção, destaca-se que se faz necessário traçar o recorte programático da análise proposta, que para ela é a definição do que se quer analisar em uma política, ou programa ou projeto (DRAIBE, 2001).

Não há unanimidade entre os autores acerca da definição de política, plano, programa e projeto. O termo programa designa uma seqüência de ações limitadas no tempo e, sobretudo definida precisamente em seus meios e seus objetivos operatórios [...], a noção de política, quanto a ela, remete principalmente a um conjunto complexo de programas, processos e regulações, concorrendo a um mesmo objetivo geral (KRESSLER, 1998).

É muito conhecida a terminologia utilizada pelo Banco Interamericano de DesenvolvimentoBIRD ou Banco Mundial, cuja diferenciação considera a amplitude do campo econômico abrangido. O plano se aplica a economia como um todo (nacional, regional), o programa se refere à orientação de um setor, enquanto o projeto designa a orientação de um empreendimento específico Essa terminologia ajuda a esclarecer esses instrumentos, mas é preciso também admitir que muitos autores utilizam os termos como sinônimos, um projeto para o Brasil, por exemplo, pode dizer respeito a um plano (MIGLIOLI, 1983).

Após esses esclarecimentos conceituais, finaliza-se essa seção, destacando a importância da avaliação para o processo de gestão das políticas públicas. Dentre outros aspectos, na área de turismo, o processo de avaliação se faz importante pela possibilidade de contribuir no acompanhamento das ações e realinhamento das decisões e opções políticas programadas. Carvalho (2001, p. 63) observa que é preciso mudar a concepção de que a avaliação é 


\section{TURISMO EM ANÁLISE}

sinônimo de fiscalização e auditoria e aprendê-la "como um dos processos indispensáveis na melhoria das decisões e ações no campo social".

\section{Procedimentos Metodológicos}

Analisar a produção científica de uma área ou temática utilizando a bibliometria não é uma experiência nova. Observam-se na literatura diversos estudos com o objetivo de levantar indicadores de produções acadêmicas, como os de Aragão e Oliveira (2007) e Igarashi, Ensslin e Todesco (2007).

A pesquisa realizada sobre a produção científica de turismo ora apresentada, caracteriza-se, quanto à natureza, como descritiva. Sendo a análise de dados realizada de forma quantitativa $\mathrm{e}$ qualitativa. Segundo, Caldas e Tinoco $(2004$, p. 102) “a principal função das citações é fornecer ao leitor referências importantes sobre o campo de estudo em questão." No caso deste trabalho, o campo de estudo é o da avaliação de políticas públicas de turismo e as autoras têm como objetivo identificar estudos, autores e obras de referência sobre a temática e metodologias utilizadas. O critério para seleção dos periódicos foi o seguinte: todos os periódicos que incluem a palavra turismo no seu título e que tenham obtido avaliação e classificação no Qualis da CAPES de pelo menos B4, independente da área.

Para a elaboração deste levantamento foi criada uma base com os dados sobre os artigos e as referências de todos os artigos publicados nos periódicos nos últimos oito anos (2003-2010). O corte temporal foi feito tomando como referência o início do Plano Nacional de Turismo, período de maior ênfase da atividade no país. O critério de seleção dos artigos inicialmente foi a existência de alguma das palavras no título dos artigos: avaliação, análise, programa, política, projeto, indicadores, efeitos ou impacto. Sendo assim, foram selecionados 57 artigos preliminarmente (Momento 1). Após a leitura dos resumos dos 57 artigos selecionados, foram desprezados 35 artigos por não tratarem de intervenções públicas planejadas ou não planejadas (Momento 2). Após a leitura completa dos 22 artigos restantes, foi observado que 13 não tratavam de políticas, programas ou projetos públicos na área de turismo, mas estavam ligados a gestão privada de empreendimentos turísticos. (Momento 3). 
Tabela 1 - Periódicos selecionados para a pesquisa

\begin{tabular}{|c|c|c|c|c|c|c|}
\hline \multirow{2}{*}{ PERIÓDICO } & \multirow{2}{*}{$\begin{array}{l}\text { ESTRATO } \\
\text { QUALIS } \\
\end{array}$} & \multirow{2}{*}{ ÁREA $^{4}$} & \multirow{2}{*}{$\begin{array}{c}\text { PERÍODO } \\
\text { DISPONÍVEL }\end{array}$} & \multicolumn{3}{|c|}{ ARTIGOS } \\
\hline & & & & (1) & $(2)$ & (3) \\
\hline Estudios y Perspectivas en Turismo & $\mathrm{B} 1$ & 1 & $2003-2008$ & $8^{6}$ & 0 & 0 \\
\hline $\begin{array}{c}\text { Pasos - Revista de Turismo y } \\
\text { Patrimonio Cultural }\end{array}$ & B1 & 1 & 2003-2010 & 10 & 4 & 1 \\
\hline Cuadernos de Turismo & B2 & 1 & $2003-2009$ & 3 & 1 & 0 \\
\hline Revista Turismo em Análise & $\mathrm{B} 2$ & 1 & ND & ND & ND & $\mathrm{ND}$ \\
\hline Turismo. Visão e Ação & B3 & 1 & $2003-2010$ & 6 & 5 & 3 \\
\hline $\begin{array}{l}\text { Revista Acadêmica Observatório de } \\
\text { Inovação do Turismo }\end{array}$ & B4 & 1 & 2006-2009 & 7 & 3 & 1 \\
\hline Caderno Virtual de Turismo (UFRJ) & $\mathrm{B} 4$ & 1 & $2003-2010$ & 20 & 9 & 4 \\
\hline $\begin{array}{c}\text { Anais - Encontro Nacional de Turismo } \\
\text { com Base Local }\end{array}$ & B4 & 2 & ND & ND & ND & ND \\
\hline Revista Brasileira de Ecoturismo & B4 & 2 & $2008-2010$ & 3 & 0 & 0 \\
\hline $\begin{array}{c}\text { Revista Brasileira de Pesquisa em } \\
\text { Turismo } \\
\end{array}$ & B4 & 3 & $2007-2008$ & 0 & 0 & 0 \\
\hline $\begin{array}{c}\text { Revista Eletrônica de Turismo } \\
\text { Cultural (USP) }\end{array}$ & B4 & 4 & ND & ND & ND & ND \\
\hline & & & TOTAL & 57 & 22 & 9 \\
\hline
\end{tabular}

Fonte: elaboração própria baseado em Capes (2010) e nos sites dos periódicos.

ND significa que a revista não estava disponível em meio eletrônico para consulta.

A primeira etapa da pesquisa teve como resultado a alimentação da planilha no Excel com os dados dos artigos separados em campos distintos para posterior tratamento quantitativo e indicações de autores, instituições e obras de referências utilizadas pelos mesmos. A segunda etapa consistiu de uma profundidade maior de análise através da técnica de análise de conteúdo, entendida como um procedimento sistemático para descrever o conteúdo de comunicações (MERRIAM, 1998, p. 123). Os 9 artigos selecionados $^{7}$ foram trabalhados de forma a verificar aplicações das categorias teóricas descritas na seção anterior.

\footnotetext{
${ }^{4} 1$ - Administração, ciências contábeis e turismo, 2 - Geografia, 3 - Interdisciplinar, 4 - Educação.

${ }^{5}$ Período de coleta encerrado em junho de 2010.

${ }^{6}$ Um artigo não estava disponível para download.

${ }^{7}$ Artigos analisados:

COSTA, Jean Henrique. Políticas públicas, turismo e emprego no litoral potiguar. Caderno Virtual de Turismo. v. 8, n. 2, p. 115-129, 2008.

DELGADO, Anna Karenina Chaves. As ações do PRODETUR/NE I e suas implicações para o desenvolvimento da Paraíba com base no turismo. Caderno Virtual de Turismo. v, 9, n. 3, p. 32-43, 2009.

MUNIZ, Caroline Feitosa Gama; SANTOS, Saulo Ribeiro dos. A implantação do macroprograma de comunicação do plano integral de desenvolvimento do turismo no Maranhão: o caso do município de São Luiz (MA). Caderno Virtual de Turismo. v. 7, n. 1, p. 10-18, 2007.
} 


\section{Descrição e Análise dos Dados}

Os dados são apresentados em duas partes: uma referente às informações bibliométricas e outra referente à análise, tipologia e procedimentos de avaliação utilizados.

\subsection{Informações bibliométricas}

Conforme a tabela 1, apresentada na seção anterior, pode-se notar que os trabalhos sobre avaliação de políticas públicas na área de turismo não correspondem a um por cento ${ }^{8}$ dos trabalhos publicados nos melhores periódicos da área em língua portuguesa e espanhola, avaliados pela CAPES e dos quais se teve acesso aos artigos pelo website das revistas.

Quando analisado por periódico, como mostra a tabela 2 abaixo, percebe-se que também não se pode atribuir um periódico de referência ao tema em análise, pois mesmo na revista que mais publicou sobre o tema, a Revista Turismo: visão e ação, a temática não representa $2,2 \%$ dos artigos publicados.

SANTOS, Saulo Ribeiro dos; TEIXEIRA, Maria Gracinda Carvalho. Análise do plano de desenvolvimento turístico do estado do Maranhão: potencialidades e entraves na gestão do pólo turístico. Turismo. Visão e Ação. v. 11, n. 2, p. 218-241, 2009.

SILVA, Josilene Henriques da; MAIA, Fabiana Britto de Azevedo. O turismo no Parque Nacional do Catimbau: avaliação dos benefícios da atividade percebidos pelos moradores. Turismo. Visão e Ação. v. 10, n. 2, p. 204 220, 2008.

SILVA, Luciana Rodrigues de Morais.; ANDRADE, José Roberto de Lima. Programa de regionalização do turismo e sua aplicação em comunidades autóctones: o caso do Poço Redondo e Canindé do São Francisco - SE. Caderno Virtual de Turismo. v. 8, n. 2, p. 15-22, 2008.

SILVA, Tatiana Amaral; ÁVILA, Marcos Aurélio. Turismo sexual e exploração sexual infantil: uma análise da atuação do programa Sentinela, em Ilhéus. Pasos - Revista de Turismo y Patrimonio Cultural. v. 8, n. 1, p. 185 193, 2010.

TAVEIRA, Marcelo da Silva; FONSECA, Maria Aparecida Pontes da. O modelo turístico potiguar e seus efeitos sócio-espaciais nas comunidades litorâneas. Observatório de Inovação do Turismo. v. 4, n. 4, 2009.

TRENTIN, Fábia; SANSOLO, Davis Gruber. Políticas públicas de turismo e indicadores de sustentabilidade ambiental: um estudo sobre Bonito/MG. Turismo. Visão e Ação. v. 8, n. 1, p. 61-74, 2006.

${ }^{8}$ De um total de 959 artigos publicados pelos periódicos no período selecionado. 
Tabela 2 - Produção acadêmica sobre avaliação de políticas públicas nos periódicos analisados

\begin{tabular}{cccc}
\hline PERIÓDICO & $\begin{array}{c}\text { ARTIGOS } \\
\text { PUBLICADOS }\end{array}$ & $\begin{array}{c}\text { TRABALHOS } \\
\text { SOBRE } \\
\text { AVALIAÇÃo }\end{array}$ & $\begin{array}{c}\text { \% DE ARTIGOS } \\
\text { SOBRE AVALIAÇÃo }\end{array}$ \\
\hline $\begin{array}{c}\text { Pasos - Revista de Turismo y } \\
\text { Patrimonio Cultural }\end{array}$ & 211 & 1 & $0,47 \%$ \\
\hline Turismo. Visão e Ação & 142 & 3 & $2,11 \%$ \\
\hline $\begin{array}{c}\text { Revista Acadêmica Observatório de } \\
\text { Inovação do Turismo }\end{array}$ & 78 & 1 & $1,28 \%$ \\
\hline Caderno Virtual de Turismo (UFRJ) & 202 & 4 & $1,98 \%$ \\
\hline TOTAL & 633 & 9 & $1,42 \%$ \\
\hline
\end{tabular}

Fonte: periódicos da pesquisa

Também não foram identificadas instituições e autores de referência, já que as universidades apareceram apenas uma vez nos artigos analisados, com exceção da Universidade Federal do Maranhão com dois artigos publicados pelo professor Saulo Ribeiro dos Santos, como pode ser observado no quadro 1, abaixo:

\begin{tabular}{|c|c|}
\hline AUTORES & UNIVERSIDADES* \\
\hline Anna Karenina Chaves Delgado & Universidade de Brasília \\
\hline $\begin{array}{l}\text { Caroline Feitosa Gama Muniz } \\
\text { Saulo Ribeiro dos Santos }\end{array}$ & $\begin{array}{l}\text { (não possui currículo na plataforma Lattes) } \\
\text { Universidade Federal do Maranhão }\end{array}$ \\
\hline $\begin{array}{l}\text { Fábia Trentin } \\
\text { Davis Gruber Sansolo }\end{array}$ & $\begin{array}{l}\text { Universidade Federal Fluminense } \\
\text { Universidade Anhembi Morumbi }\end{array}$ \\
\hline Jean Henrique Costa & Universidade do Estado do Rio Grande do Norte \\
\hline $\begin{array}{l}\text { Josilene Henriques da Silva } \\
\text { Fabiana Britto de Azevedo Maia }\end{array}$ & $\begin{array}{l}\text { Universidade Federal de Pernambuco } \\
\text { Universidade Federal de Pernambuco }\end{array}$ \\
\hline $\begin{array}{l}\text { Luciana Rodrigues de Morais e Silva } \\
\text { José Roberto de Lima Andrade }\end{array}$ & $\begin{array}{l}\text { Universidade Federal do Sergipe - Universidade do Tiradentes } \\
\text { Universidade Federal do Sergipe }\end{array}$ \\
\hline $\begin{array}{l}\text { Marcelo da Silva Taveira } \\
\text { Maria Aparecida Pontes da Fonseca }\end{array}$ & $\begin{array}{l}\text { Instituto Federal do Rio Grande do Norte } \\
\text { Universidade Federal do Rio Grande do Norte }\end{array}$ \\
\hline$\frac{\text { Saulo Ribeiro dos Santos }}{\text { Maria Gracinda Carvalho Teixeira }}$ & $\begin{array}{l}\text { Universidade Federal do Maranhão } \\
\text { Universidade Federal do Maranhão }\end{array}$ \\
\hline $\begin{array}{l}\text { Tatiana Amaral Silva } \\
\text { Marcos Aurélio Ávila }\end{array}$ & $\begin{array}{l}\text { Universidade Estadual de Santa Cruz } \\
\text { Universidade Estadual de Santa Cruz }\end{array}$ \\
\hline
\end{tabular}

Fonte: dados da pesquisa

* Instituição de origem no momento da publicação do artigo. 
Em relação à utilização de referências sobre políticas públicas e avaliação das mesmas nota-se uma lacuna de textos conceituais sobre a área, ou seja, artigos são produzidos e publicados sem a utilização e embasamento de autores que estudam o assunto, seja na área específica de turismo ou não. $\mathrm{O}$ fato se torna mais preocupante quando não são percebidos autores de referências, com idéias já consolidadas e reconhecidas na academia sobre os temas em questão e uma ausência completa da literatura específica de avaliação. As obras citadas com o título de políticas púbicas, mas não de forma recorrente, foram as seguintes:

Tabela 3 - Referências específicas utilizadas e freqüência de uso

\begin{tabular}{|c|c|}
\hline REFERÊNCIA & No. CITAÇÕES \\
\hline $\begin{array}{l}\text { CRUZ, Rita de Cássia Ariza da. Política de turismo e território. 2. ed. São Paulo: } \\
\text { Contexto, } 2001 .\end{array}$ & 3 \\
\hline $\begin{array}{l}\text { FONSECA, Maria Aparecida Pontes. Espaço, políticas de turismo e } \\
\text { competitividade. Natal-RN: EDUFRN, } 2005 .\end{array}$ & 2 \\
\hline $\begin{array}{l}\text { BUCCI, Maria Paula Dallari. Políticas Públicas: reflexões sobre o conceito jurídico. } \\
\text { São Paulo: Saraiva, } 2006 .\end{array}$ & 1 \\
\hline $\begin{array}{l}\text { MIRANDA, Maria Inês Ferreira de. Políticas Públicas: reflexões para crianças e } \\
\text { adolescentes. Goiânia: AB, } 2001 \text {. }\end{array}$ & 1 \\
\hline $\begin{array}{l}\text { MERICO, K. F. K. Políticas Públicas para a sustentabilidade. In: VIANA, G.; SILVA, } \\
\text { M; DINIZ, N (orgs.) O desafio da sustentabilidade. São Paulo: editora Fundação } \\
\text { Perseu Abramo, 2001, p. 251-262. }\end{array}$ & 1 \\
\hline $\begin{array}{l}\text { BARBOSA, L.G. M.; ZAMOT, F. S. Políticas públicas para o desenvolvimento do } \\
\text { turismo: o caso do município de Rio das Ostras. In: BARBOSA, L. G. M.; ZOUAIN, } \\
\text { D., M. (Orgs.) Gestão em turismo e hotelaria: experiências públicas e privadas. } \\
\text { São Paulo: Aleph, } 2004 \text {. }\end{array}$ & 1 \\
\hline $\begin{array}{l}\text { OLIVEIRA, J. A. P. de. Repensando Políticas Públicas: Por que freqüentemente } \\
\text { falhamos no planejamento? In: ENCONTRO NACIONAL DE PÓS-GRADUAÇÃO } \\
\text { EM ADMINISTRAÇÃO, 29, Brasília. Anais... Brasília: EnANPAD, 2005. } 1 \text { CD. }\end{array}$ & 1 \\
\hline $\begin{array}{l}\text { OLIVEIRA, J. A. P. de. Implementation of environmental policies in developing } \\
\text { countries. Albany: State University of New York Press, } 2008 .\end{array}$ & 1 \\
\hline $\begin{array}{l}\text { COSTA, Jean Henrique. Trabalhadores de verão: Políticas públicas, turismo e } \\
\text { emprego no litoral potiguar. Natal: UFRN, 2007. Dissertação (Mestrado) - Centro de } \\
\text { Ciências Humanas, Letras e Artes, Universidade Federal do Rio Grande do Norte, } \\
\text { Natal, } 2007 .\end{array}$ & 1 \\
\hline $\begin{array}{l}\text { CRUZ, Rita de Cássia Ariza da. Políticas de Turismo e construção do espaço turístico } \\
\text { litorâneo do Nordeste do Brasil. In: LEMOS, A. I. G (org.). Turismo: impactos } \\
\text { socioeconômicos. São Paulo: Hucitec, 1999. }\end{array}$ & 1 \\
\hline $\begin{array}{l}\text { TAVEIRA, Marcelo da Silva. Políticas de turismo e comunidade local no litoral } \\
\text { potiguar. Natal: UFRN, 2008. Dissertação (Mestrado) - Centro de Ciências } \\
\text { Humanas, Letras e Artes, Universidade Federal do Rio Grande do Norte, Natal, } 2008 .\end{array}$ & 1 \\
\hline $\begin{array}{l}\text { CAVALCANTI, K. B. Estado e política de turismo: o caso da Via Costeira da cidade } \\
\text { de Natal. Dissertação (mestrado em Administração). Nata: Centro de Ciências Sociais } \\
\text { Aplicadas, Universidade Federal do Rio Grande do Norte, Natal, } 1993\end{array}$ & 1 \\
\hline
\end{tabular}




\subsection{Resultados da análise dos artigos à luz das categorias selecionadas}

Para analisar os artigos foram as seguintes categorias de análise: tempo e lugar da análise, recorte programático, referências de políticas públicas e de avaliação utilizadas, unidade de análise, dimensões enfocadas, tipologia de avaliação, utilização de indicadores e procedimentos metodológicos.

Os artigos expuseram de forma clara o recorte programático e o lugar utilizado pelos mesmos. Oito artigos analisaram programas, os quais são especificados a seguir: (Programa Sentinela, em Ilhéus; Plano Maior, no Maranhão); Prodetur NE/I, nos municípios da região metropolitana de Natal, dois artigos; e Prodetur NE/I, no estado da Paraíba; Programa de Regionalização do Turismo, em Poço Redondo e Canindé do São Francisco, em Sergipe; Programa de Implementação das Unidades de Conservação, em Buíque, Pernambuco). O último artigo tratou da política de turismo, em Bonito/MG.

Com exceção de um que se voltou para a análise do Plano Maior do Maranhão, todos os outros realizaram suas análises tendo como unidade de análise a localidade, seja um município ou um conjunto desses que foram alvo da política ou analisado. As dimensões de análise mais freqüentes foram as econômicas e sociais, através da investigação de impactos referentes ao emprego e renda, mas também foram identificadas as dimensões ambientais e política. Todas as análises foram realizadas mediante consulta às pessoas externas à execução dos programas, geralmente ligadas a programas de pós-graduação de Universidades da referida região. Quanto à dimensão temporal, todos os artigos se concentraram na avaliação ex post, quando focam a análise do desenvolvimento ou nos produtos verificados com a implementação do programa. Avaliações ex post, são feitas durante ou após a realização do projeto, quando o programa alcançou seu pleno desenvolvimento e meses ou anos depois de finalizada a execução.

A ausência de literatura específica sobre avaliação tem implicações nos procedimentos metodológicos utilizados de forma a responder as perguntas de pesquisa formuladas nos estudos. O uso de indicadores ou variáveis de análise, ou pelo menos sua explicitação nos textos, foram realizadas apenas nos artigos que se referiram aos programas: Sentinela, em 


\section{TURISMO EM ANÁLISE}

Ilhéus, Programa Maior, no Maranhão em apenas um dos dois artigos estudados; e PRODETUR NE/I, na Paraíba; e a política pública de turismo em Bonito/MG. No entanto, sem aprofundamento ou maiores detalhes sobre os mesmos.

Talvez, por falta de definição de indicadores e análise mais sistemática, nos artigos, algumas conclusões foram apresentadas de forma superficial e sem demonstrar que os efeitos/resultados verificados tenham sido devidos a implementação do programa estudado e não de outro. Dois dos artigos que se propuseram a investigar os resultados/efeitos de programas junto a população chegaram a conclusões semelhantes: "após a implementação da ação, houve um aumento do número de empregos, mas seguida de concentração de renda, no período analisado". Conclusões como essas suscitam questionamentos como os que se seguem: como o autor pode assegurar que esses resultados estão apenas atrelados aos investimentos, ações ou omissões governamentais na área do turismo? Outros fatores não poderiam contribuir ou influenciar os resultados?

Diversos artigos também relataram fatos, mas não deixaram explicitado o nível de ocorrência dos mesmos. Por exemplo, frases como as seguintes foram muito comuns: "os benefícios advindos atingem um número incipiente de moradores" ou "o grau de conhecimento acerca dos projetos dessas duas comunidades é ínfimo". Portanto, é pertinente o questionamento sobre estas assertivas presentes nos artigos citados: o que eles consideram como incipientes? O que é definido como um grau de conhecimento ínfimo? Faltou, durante as leituras, uma melhor definição do que estava sendo objeto de investigação e quais variáveis e indicadores estavam sendo utilizados.

Outra dificuldade ocorreu devido à utilização dos construtos de resultados, impactos e efeitos como se fossem sinônimos. Informava-se que iriam ser investigados os efeitos, quando, na realidade, o que se deixava claro eram os resultados imediatos, por exemplo. Também se percebe um certo descuido por normas metodológicas ou que não foram devidamente explicitadas na maioria dos artigos selecionados. Os artigos analisados, e não se tratam apenas dos 9 finais, mas os 23 que foram lidos integralmente, cometeram equívocos do tipo: 
a) descreveram que utilizaram a técnica de entrevistas para a coleta de dados, no entanto, não especificaram quais os públicos-alvo investigados, nem os critérios de seleção dos informantes-chave;

b) informaram ter utilizado uma amostra não probabilística, intencional, e com o quantitativo muito inferior ao tamanho da amostra recomendado para obter representatividade estatística e o texto fazia inferências para toda a população;

c) ressaltaram ter utilizado documentos, mas não os especificaram;

d) não informaram o período de coleta de dados;

e) não informaram quais técnicas de análise de dados foram utilizadas;

f) quando destacaram as técnicas utilizadas, por exemplo, análise de conteúdo, não explicaram as categorias analíticas.

A falta dessas informações prejudicou a análise dos artigos e possíveis replicações do mesmo em outros contextos de forma a confrontar ou confirmar resultados encontrados anteriormente.

\section{Conclusões}

Este trabalho teve como objetivo ampliar o conhecimento sobre avaliação de políticas públicas de turismo a partir de um estudo bibliométrico nos periódicos especializados avaliados pela CAPES com conceito igual ou superior a B4, bem como apontar perspectivas de análise para aprofundamentos posteriores.

A pesquisa realizada para fins do presente artigo comprovou que é pequena a produção científica sobre a avaliação de políticas públicas nos periódicos especializados em turismo no Brasil. Observou também que os artigos examinados apresentaram, em sua maioria, fragilidades e inconsistências metodológicas quanto aos métodos e categorias analíticas utilizadas, o que comprometeu a percepção e o exame dos resultados por eles apresentados, 


\section{TURISMO EM ANÁLISE}

que foram mais gerais e abstratos que específicos e precisos, dificultando ou impossibilitando a replicação das pesquisas em outros contextos.

Constatou também o uso restrito da literatura consagrada sobre avaliação de políticas públicas. Esse aspecto foi, sem duvida, um elemento dificultador, pois a falta de clareza sobre o tipo de avaliação que se pretendia fazer, se refletiu na indefinição de critérios para aferição de resultados, efeitos e impactos passíveis de serem apreendidos ou mensurados. Dessa forma, deixou-se também de contemplar as limitações dos estudos, bem como os efeitos de ligações entre as políticas de turismo e outras políticas públicas.

Esses aspectos demonstram que a avaliação de políticas públicas em turismo é um campo de estudo vasto que tem ainda um longo caminho a percorrer, campo necessário que lhe seja dado prioridade pelos estudiosos, sobretudo devido à importância que a atividade turística tem apresentado em nosso país e pelo papel estratégico que as políticas públicas têm desempenhado no desenvolvimento da área, exigindo assim, conhecimento mais detalhado e específico de métodos e técnicas de pesquisas, afinados com a avaliação de planos, programas e projetos do setor, que alcança abrangência territorial diversificada e níveis de atuação em âmbito federal, estadual e municipal, além de recortes regionais.

\section{Referências}

AGUILAR, Maria José; ANDER-EGG, Ezequiel. Avaliação de serviços e programas sociais. Petrópolis, RJ: Vozes, 1994.

ARAGÃO, Lindenberg Araújo; OLIVEIRA, Oderlene Vieira de. Visão baseada em recursos e capacidades dinâmicas no contexto brasileiro. A produção e a evolução acadêmica em dez anos de contribuições. In: 31 ENCONTRO NACIONAL DE PROGRAMAS DE PÓS-GRADUAÇÃO EM ADMINISTRAÇÃO, 2007, Rio de Janeiro. Anais... Rio de Janeiro: ANPAD, 2007. 1 CD ROM.

BROHMAN, John. New directions in tourism for third world development. Annals of Tourism Research. v. 23, n. 1, p. 48-70, 1996.

CAIDEN, Gerald E.; CAIDEN, Naomi J. Enfoques y lineamentos para el seguimiento, la medición y la evaluación del desempeño en programas del sector público. Revista do Serviço Público, Brasília, ano 52, n. 4, p. 78-101, out./dez. 2001.

CALDAS M. P.; TINOCO, T. Pesquisa em gestão de recursos humanos nos anos 1990: um estudo bibliométrico. Revista de Administração de Empresas, v. 44, n. 3, p.100-114, 2004. 
CAPES - Coordenação de Aperfeiçoamento de Pessoal de Nível Superior. Web-Qualis. Avaliação de Periódicos. Disponível em: <http://qualis.capes.gov.br/webqualis/>. Acesso em 26 jun. 2010.

CARVAlHO, Maria do Carmo Brant de. Avaliação de projetos sociais. In: ÁvILA, Célia M. de (coord.). Gestão de projetos sociais. São Paulo: AAPCS - Associação de Apoio ao Programa Capacitação Solidária, 2001. p. 61-92.

DRAIBE, Sônia Miriam. Avaliação de implementação: esboço de uma metodologia de trabalho em políticas públicas. In: BARREIRA, Maria Cecília Roxo Nobre; Maria do Carmo Brant de CARVALHO (Org.). Tendências e perspectivas na avaliação de políticas e programas sociais. São Paulo: IEE/PUC-SP, 2001.

FARIA, Regina. Avaliação de programas sociais: evolução e tendências. In: RICO, Elizabeth Melo (Org.). Avaliação de políticas sociais: uma questão em debate. São Paulo: Cortez: Instituto de Estudos Especiais, 2007. p. 41-49.

FIGUEIREDO, Marcos Faria; FIGUEIREDO, Argelina Maria Cheibub. Avaliação política e avaliação de políticas. Análise \& Conjuntura, v.1, n. 3, Belo Horizonte, set./dez. 1986.

GOELDNER, Charles R.; RITCHIE, J. R. Brent; McINTOSH, Robert W. Políticas de turismo: estrutura, conteúdo e processo. In: 2002. p. 293-312. Turismo: princípios, práticas e filosofia. Porto Alegre,

IGARASHI, Deisy Cristina Corrêa; ENSSLIN, Sandra Rolim; IGARASHI, Wagner, TODESCO, José Leomar; ENSSLIN, Leonardo. Avaliação/Ensino/Pós-graduação no contexto brasileiro: uma investigação sobre a produção científica constante na Scientific Electronic Library Online, no período entre 1974 e 2007. In: 31 ENCONTRO NACIONAL DE PROGRAMAS DE PÓS-GRADUAÇÃO EM ADMINISTRAÇÃO, 2007, Rio de Janeiro. Anais... Rio de Janeiro: ANPAD, 2007. 1 CD ROM.

JANNUZZI, Paulo de Martino. Considerações sobre o uso, mau uso e abuso dos indicadores sociais na formulação e avaliação de políticas públicas municipais. Revista de Administração Pública, Rio de Janeiro, v. 36, n. 1, p. 51-72, jan./fev. 2002.

Indicadores para diagnóstico, monitoramento e avaliação de programas sociais no Brasil.

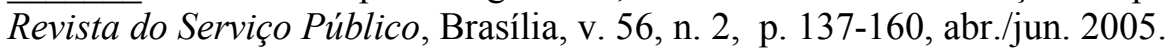

. Repensando a prática de uso de indicadores sociais na formulação e avaliação de políticas públicas municipais. In: KEINERT, Tânia Margarete Mezzomo; KARRUZ, Ana Paula (org.). Qualidade de Vida: observatórios, experiências e metodologias. São Paulo: Annablume: Fapesp, 2002a. p. 53-72.

KRESSLER, Marie Christine, Avant-Propos. In KRESSLER, Marie Christine; LASCOUMES, Pierre; SETBOM, Michel; THOENIG Jean Claude(orgs.). Evaluation des politiques publiques. Paris: L'Harmattan, 1998, p.1-5.

MERRIAM, S. B. Case Studies as Qualitative Research. In: study applications in education. San Francisco: Jossey-Bass, 1998. p. 26-43.

MIGLIOLI, Jorge. Introdução ao planejamento econômico. São Paulo: Ed. Brasiliense, 1983.

MINAYO, Maria Cecília de Souza Introdução: conceito de avaliação por triangulação de métodos. In:

; ASSIS, Simone Gonçalves de; SOUZA, Edinilsa Ramos de (orgs.). Avaliação por triangulação de métodos: abordagem de programas sociais. Rio de Janeiro: Fiocruz, 2005. p. 19-51. 
PINTO, Ana Maria Rezende. O fetichismo da avaliação. Análise \& Conjuntura, Belo Horizonte, v. 1, n.2, p. 73-92, mai/ago. 1986.

Recebido em: 13/04/2011

Aprovado em: 16/08/2011 